\title{
Central aortic valve coaptation area during diastole as seen by 64-multidetector computed tomography (MDCT)
}

\author{
Irfan Zeb • Song Shou Mao • Yasmin S. Hamirani • Sameer Raina • \\ Jigar Kadakia $\cdot$ Sameh Elamir $\cdot$ Matthew J. Budoff
}

Received: 27 January 2010/ Accepted: 10 May 2010/Published online: 26 May 2010

(C) The Author(s) 2010. This article is published with open access at Springerlink.com

\begin{abstract}
As multiple new procedures now require better visualization of the aortic valve, we sought to better define the central aortic valve coaptation area seen during diastole on multi-detector row cardiac computed tomography (MDCT). 64-MDCT images of 384 symptomatic consecutive patients referred for coronary artery disease evaluation were included in the study. Planimetric measurements of this area were performed on cross-sectional views of the aortic valve at $75 \%$ phase of the cardiac cycle. Planimetric measurement of central regurgitation orifice area (ROA) seen in patients with aortic regurgitation and Hounsfield units of the central aortic valve coaptation area were performed. Mean area of the central aortic valve coaptation area was $5.34 \pm 5.19 \mathrm{~mm}^{2}$ and Hounsfield units in this area were $123.69 \pm 31.31$ HU. The aortic valve coaptation area $\left(\mathrm{mm}^{2}\right)$ measurement in patients without AR was: $4.90 \pm 0.17$ and in patients with AR: $10.53 \pm 0.26(P=<0.05)$. On Bland-Altman analysis a very good correlation between central aortic valve coaptation area and central ROA was found $(r=0.80, P=<0.001)$. Central aortic valve coaptation area is a central area
\end{abstract}

I. Zeb - S. S. Mao - Y. S. Hamirani - S. Raina ·

J. Kadakia · S. Elamir · M. J. Budoff $(\bowtie)$

Los Angeles Biomedical Research Institute

at Harbor-UCLA, 1124 West Carson Street,

Torrance, CA 90502, USA

e-mail: mbudoff@labiomed.org present at the coaptation of nodules of arantius of aortic cusps during diastole; it is incompetent and increased in size in patients with aortic regurgitation.

Keywords Aortic regurgitation .

Central aortic valve coaptation area .

64-Multidetector computed tomography
Abbreviations
MDCT Multidetector computed tomography
AR Aortic regurgitation
ROA Regurgitation orifice area
TTE Trans-thoracic echocardiography
HU Hounsfield units
CTA Computed tomography angiography

\section{Introduction}

Aortic regurgitation (AR) is characterized by the diastolic reflux of blood flow from the aorta to the left ventricle due to incomplete coaptation of the aortic valve. The overall prevalence of AR was $4.9 \%$ in the Framingham Heart Study [1] and 10\% in the Strong Heart Study [2]. There are studies that have been performed to visualize the $\mathrm{AR}$ on the multi-detector computed tomography (MDCT) scans [3-6]. In this study, we intend to mention a part of the aortic valve that becomes incompetent in aortic regurgitation and is visible on the MDCT scans. Normally, the aortic 
valve consists of three leaflets and three sinuses. The leaflets are mobile parts of the aortic valve whereas the sinuses are the cavities behind the leaflets. Each leaflet is attached to the aortic wall and free margin of each leaflet coaptate with each other along the line of leaflet coaptation during diastole. During systole, the leaflets are open to allow blood flow across the aortic valve from the left ventricle to the aorta. During diastole, these leaflets close to hold the column of blood in the aorta while the left ventricle is being filled during diastole. Normally, these valves are competent enough to withhold any blood flow from aorta to the left ventricle during diastole. But in aortic regurgitation, this mechanism fails and blood regurgitates across the aortic valve to the left ventricle. This is visualized as regurgitant jet on the echocardiography and as regurgitant orifice area (ROA) on multidetector computed tomography (MDCT) [3-7]. In normal tricuspid aortic valves, the leaflets coapt along the line of leaflet coaptation and become visible as hypodense area between aortic leaflets on MDCT scans. The central part of this coaptation is due to coaptation of nodules of Arantius present in middle of free margin of the aortic leaflets. We called this central area of leaflet coaptation as 'Central Aortic Valve Coaptation Area'. Normally, this area is competent and does not allow any regurgitation. In $\mathrm{AR}$, incomplete coaptation of this area can be visualized on MDCT scans as regurgitant orifice area (ROA). In this study, we looked at this central area of aortic valve coaptation, performing its planimetric measurements and also determining its Hounsfield Units to find its tissue properties.

\section{Methods}

Study population

Retrospective analysis of MDCT scans of 400 symptomatic consecutive patients whose scans were performed at our outpatient cardiac computed tomography scanner for the evaluation of coronary artery disease (Los Angeles Biomedical Research Institute at Harbor-UCLA Medical Center) were analyzed. Patients with poor image quality (columnation, streak artifacts, motion artifacts, and image noise), bicuspid aortic valves and heavy calcification of aortic valve leaflets that made the aortic valve region non- evaluable were excluded. Sixteen MDCT scans were excluded due to the above reason and 384 scans were included for evaluation of the aortic valve area.

\section{CT image acquisition}

Study subjects underwent MDCT angiography. All MDCT scans were performed with 64-detector row Lightspeed VCT scanner (General Electric Healthcare, Milwaukee, Wisconsin). All patients were in normal sinus rhythm at the time of the MDCT scan. Individuals presenting with baseline heart rates $>65$ beats per minute (bpm) were administered oral beta-blocker therapy as the preferred method for slowing down the heart rate. IV administration was allowed in the protocol, using intravenous metoprolol at $5 \mathrm{mg}$ increments to a total possible dose of $40 \mathrm{mg}$ in order to achieve a resting heart rate $<65 \mathrm{bpm}$. Following a scout radiograph of the chest (anteroposterior and lateral), a timing bolus (using $10 \mathrm{cc}$ of contrast) was performed to detect time to reach optimal contrast opacification in the axial image at a level immediately superior to the ostium of the left main artery. Nitroglycerin $0.4 \mathrm{mg}$ sublingual was administered immediately prior to contrast injection. During MDCT image acquisition, $60 \mathrm{cc}$ iodinated contrast was injected utilizing a triple-phase contrast protocol: 40 cc iodixanol, followed by $40 \mathrm{cc}$ of a 50:50 mixture of iodixanol and saline, followed by a $50 \mathrm{cc}$ saline flush.

The scan parameters were $64 \times 0.625 \mathrm{~mm}$ collimation, tube voltage $100-120 \mathrm{KVp}$, and effective current $350-780 \mathrm{~mA}$. Images are acquired using retrospective and prospective ECG triggering. Prospective protocol was utilized, whenever possible, to reduce radiation exposure. After scan completion, reconstruction of MDCT scans was performed at 5$95 \%$ using $10 \%$ increment on retrospective scans and $70-80 \%$ using $5 \%$ interval on prospective scans. For measurement purpose, only $75 \%$ phase of the R-R interval was utilized. This particular phase was utilized for measurement because this phase is available on both prospective and retrospective scans and it has been shown to be an optimum phase for coronary artery disease evaluation [8].

\section{MDCT measurements}

Central aortic valve coaptation area is identified as circular to triangular shape area present centrally in 

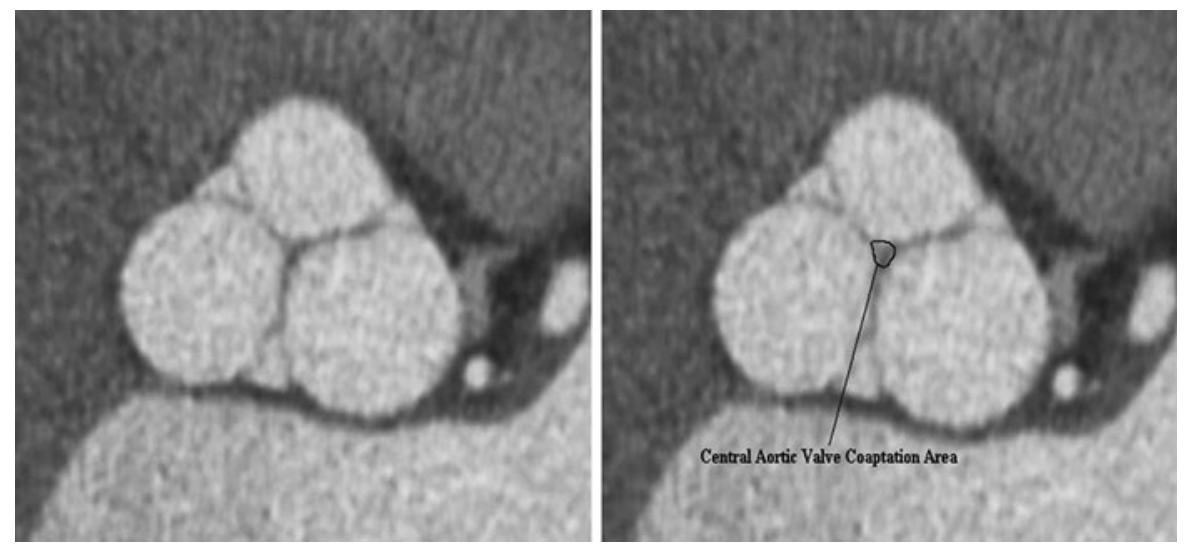

Fig. 1 Central aortic valve coaptation area as seen on MDCT scan

the aortic valve during diastolic phases. It is visualized as a hypodense structure on contrast enhanced MDCT scans. Planimetric measurements of this area were performed on cross sectional images of the aortic valve (as shown in Fig. 1). Hounsfield units in this area were also measured. This central coaptation area is closed in normal persons, visualized as no contrast present in this area. In patients with aortic regurgitation, this area is incompetent and is visualized as regurgitant orifice area (ROA) (as shown in Fig. 2). Planimetric measurement of the ROA when observed was also performed in crosses sectional views of the aortic valve at $75 \%$ phase of the $\mathrm{R}-\mathrm{R}$ interval (Fig. 2) [5].

Continuous variables were measured using Student $t$ test and categorical variables using Chi Square test. The data was summarized as mean \pm standard deviation or numbers (percentages). Bland-Altman correlation was utilized for correlation between central aortic valve coaptation area and central ROA. The data was analyzed using commercially available statistical software STATA (version 10, College Station, TX).

\section{Results}

General characteristics of the patient population are given in the Table 1. Mean area of the central aortic valve coaptation area on planimetric measurement was $5.34 \pm 5.19 \mathrm{~mm}^{2}$. Mean Hounsfield units in this area were $123.69 \pm 31.31 \mathrm{HU}$. Incomplete co-aptation of the aortic valve cusps as ROA was noticed on 29 patients. We followed trans-thoracic echocardiography (TTE) reports on these 29 patients and all of them were found to have aortic regurgitation of various
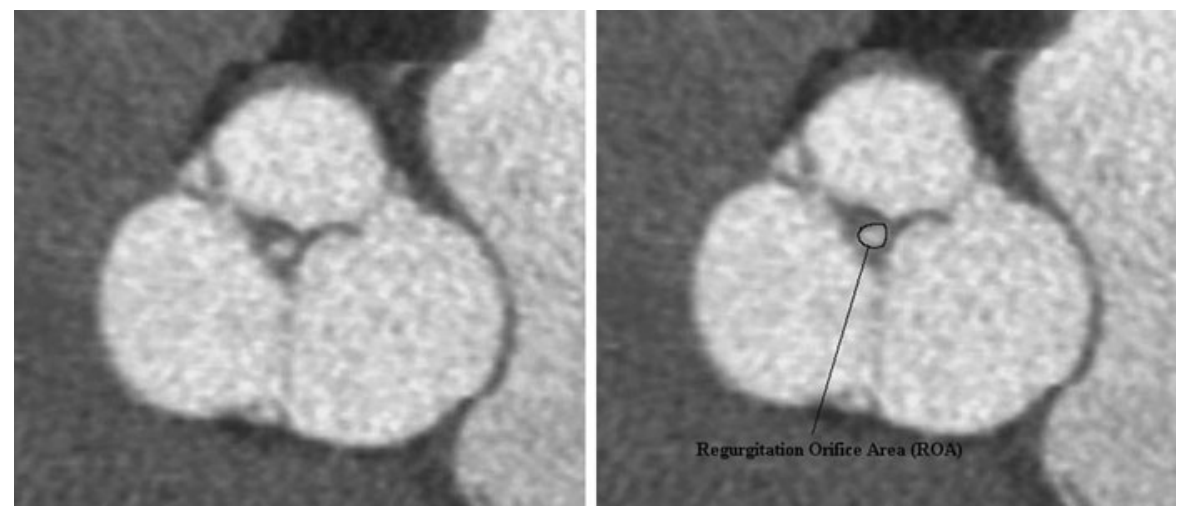

Fig. 2 Figure showing regurgitant orifice area (ROA) in patient with aortic regurgitation 
Table 1 General characteristics of patient population

\begin{tabular}{ll}
\hline Population characteristics $($ No. $=384)$ & $\begin{array}{l}\text { Mean } \pm \text { SD or No. } \\
(\%)\end{array}$ \\
\hline Age (years) & $60.37 \pm 12.86$ \\
Males & $257(67.3)$ \\
Hypertension & $108(43.03 \%)$ \\
Diabetes mellitus & $48(19.43 \%)$ \\
$\begin{array}{l}\text { Hyperlipidemia } \\
\text { Family history of CAD }\end{array}$ & $88(35.48 \%)$ \\
Central aortic valve coaptation area & $113(49.78 \%)$ \\
$\quad\left(m m^{2}\right)$ & $5.34 \pm 5.19$ \\
$\begin{array}{l}\text { Aortic valve calcium score (AU) } \\
\text { Hounsfield units of aortic pad area } \\
\quad(H U)\end{array}$ & $8.49 \pm 64.45$ \\
Regurgitant orifice area (ROA) mm ${ }^{2}$ & $6.33 \pm 8.13$ \\
\hline
\end{tabular}

severities on TTE. Mean central aortic valve coaptation area $\left(\mathrm{mm}^{2}\right)$ in patients with aortic regurgitation was $10.53 \pm 0.26$ and in patients without aortic regurgitation were $4.90 \pm 0.17$. There was correlation found between central aortic valve coaptation area and central ROA using Bland-Altman correlation which showed that with increase in ROA area, the central aortic valve coaptation area also increases in size ( $r=0.80, P=<0.001$ ) (Fig. 3). The average aortic valve calcium score in the whole cohort was $8.49 \pm 64.45$ AU.

Figure 1 shows central aortic valve coaptation area on cross-sectional views of the aortic valve as an area present in between the cusps. Figure 2 shows a patient

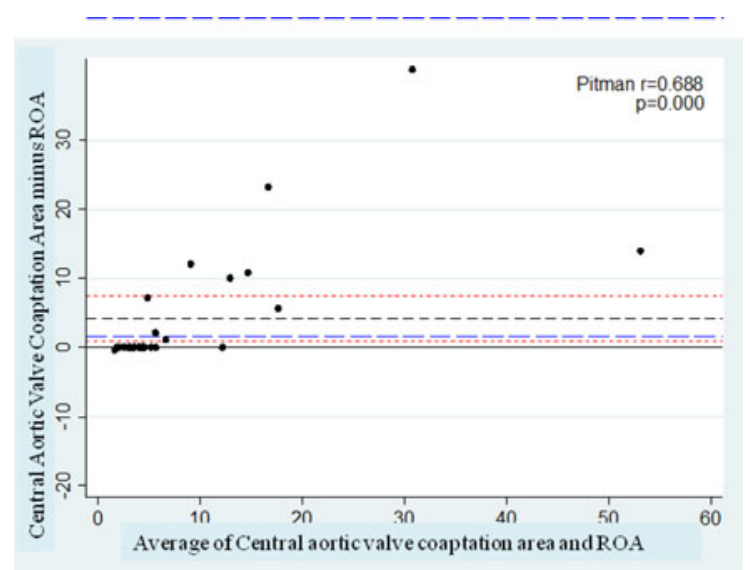

Fig. 3 Bland Altman correlation between central aortic valve coaptation area and aortic regurgitation orifice area with aortic regurgitation showing the regurgitant orifice area on cross-sectional view of the aortic valve.

\section{Discussion}

In this study, we looked at an area of the aortic valve present in the central space between the aortic cusps, 'Central Aortic Valve Coaptation Area' seen during diastole. This is an area present in normal tricuspid aortic valves. This is visualized as a circular to triangular area present centrally in between the aortic cusps during diastole. This area measures $5.34 \pm$ $5.19 \mathrm{~mm}^{2}$ on MDCT scans. This area is widely open during systole to allow normal flow of blood from the left ventricle to the aorta. During diastole, this area is normally closed and competent enough to prevent any regurgitation of blood flow from the aorta to the ventricle. In AR, we can observe incomplete coaptation of this area in case of central aortic regurgitation jet. Eccentric aortic regurgitation jets can be visualized as present in between the aortic cusps on the sides. There was a strong correlation found between increased central aortic valve coaptation area and the presence of increasing ROA due to AR. This area may not be well demarcated in patients with bicuspid aortic valves. A significant difference in the Hounsfield units was found between the contrast enhanced ROA (contrast usually 350-500 HU) and the surrounding aortic valve coaptation area $(123.69 \pm 31.31 \mathrm{HU})$. This observation will be helpful for further studies looking at the diagnostic accuracy of MDCT for AR, especially among scanners using low dose protocols, obtaining images in diastole $[8,9]$.

The limitations of our study include relatively small sample size. Transthoracic echocardiographic information was not available for all of the patients. This area may be difficult to be analyzed if there are motion artifacts, columnation artifacts, and heavy calcifications. The Hounsfield measurement in this area may be helpful but it suffers from partial volume effect. It is critical to understand that the normal aortic valve cusps have a small but defined central area visible on current cardiac CT scanners that does not represent aortic regurgitation. It is the increase in this central area along with visible ROA that represents aortic regurgitation.

Funding No outside funding was used for this study 
Open Access This article is distributed under the terms of the Creative Commons Attribution Noncommercial License which permits any noncommercial use, distribution, and reproduction in any medium, provided the original author(s) and source are credited.

\section{References}

1. Singh J, Evans J, Levy D, et al. (1999) Prevalence and clinical determinants of mitral, tricuspid, and aortic regurgitation. Am J Cardiol 83:897-902. [Erratum, Am J Cardiol 1999; 84:1143]

2. Lebowitz NE, Bella JN, Roman MJ et al (2000) Prevalence and correlates of aortic regurgitation in American Indians: the strong heart study. J Am Coll Cardiol 36(2):461-467

3. Feuchtner GM, Dichtl W, Schachner T et al (2006) Diagnostic performance of MDCT for detecting aortic valve regurgitation. AJR Am J Roentgenol 186(6):1676-1681

4. Jassal DS, Shapiro MD, Neilan TG et al (2007) 64-slice multidetector computed tomography (MDCT) for detection of aortic regurgitation and quantification of severity. Invest Radiol 42(7):507-512

5. Alkadhi H, Desbiolles L, Husmann L et al (2007) Aortic regurgitation: assessment with 64-section CT. Radiology 245(1):111-121

6. Feuchtner GM, Dichti W, Muller S et al (2008) 64-MDCT for diagnosis of aortic regurgitation in patients referred to CT coronary angiography. AJR Am J Roentgenol 191(1): W1-W7

7. Chen JJ, Jeudy J, Thorn EM, White CS (2009) Computed tomography assessment of valvular morphology, function, and disease. J Cardiovasc Comput Tomogr 3(Suppl 1): S47-S56

8. Isma'eel H, Hamirani YS, Mehrinfar R et al (2009) Optimal phase for coronary interpretations and correlation of ejection fraction using late-diastole and end-diastole imaging in cardiac computed tomography angiography: implications for prospective triggering. Int $\mathrm{J}$ Cardiovasc Imaging 25(7): 739-749

9. Gopal A, Mao SS, Karlsberg D et al (2009) Radiation reduction with prospective ECG-triggering acquisition using 64-multidetector computed tomographic angiography. Int J Cardiovasc Imaging 25(4):405-416 